\title{
Highly compact and easy-to-use optical chip interferometer with picometric performances
}

\author{
W. A. Merzouk, ${ }^{1}$ B. Cagneau, ${ }^{1}$ F. Gardillou, ${ }^{2}$ K. Hilouane,${ }^{1}$ and L. Chassagne ${ }^{1}$ \\ ${ }^{1}$ LISV, University of Versailles, 10-12 Avenue de l'Europe, 78140 Vélizy, France \\ ${ }^{2}$ Teemphotonics, 61 Chemin du Vieux Chêne, 38240 Meylan, France
}

(Received 20 February 2016; accepted 17 September 2016; published online 3 October 2016)

\begin{abstract}
In this paper, we present a compact, inexpensive, and easy-to-use optical chip interferometer based on the telecom integrated waveguide technology. The measurement evaluation is focused on the resolution and the noise level of the sensor. The power spectral density of $100 \mathrm{fm} \mathrm{Hz}^{-1 / 2}$ @ $10 \mathrm{kHz}$ is reached in static conditions. The same level is obtained with the standard Allan deviation for both short and long term measurements. Dynamic performances are also evaluated with sub-nanometer measurements made with piezoelectric systems. The potential bandwidth of the sensor is very high and is currently only limited by electronics $(250 \mathrm{kHz})$. Published by AIP Publishing. [http://dx.doi.org/10.1063/1.4963899]
\end{abstract}

\section{INTRODUCTION}

The development of sensors dedicated to nanotechnology is a full topic for several years and is still challenging. Sensing systems can still be the key elements to success in a nanotechnology process. ${ }^{1,2}$

As illustrated in Ref. 3 or Ref. 4, laboratories and industries have close relationship while working on the sensor prototypes for nanotechnologies. However, industrial partners are often more interested in products with degraded performances. It usually allows for better compactness and lower prices while ease of use, robustness, and reproducibility are preserved. On the other hand, academic partners and researchers develop new systems to constantly improve the performances.

Since a few years, our work was closely related to the development of an original sensor which has been used for specific applications like multi-scale positioning with our partner Teemphotonics.

Applications dedicated to nanoscales can relate to the manipulation of nano-objects ${ }^{5}$ but also to the positioning at nanometer level of macro-objects (sample-holder or macrotools, ${ }^{3,4}$ for example). In that case, the required performances are slightly different as multiscale capability is a concern. The stroke may be indeed in a centimetric range while the resolution is sub-nanometric. Nowadays, multiscale is still a challenge. In the literature, high resolution and high accuracy lead generally to a limited stroke lower than $1 \mathrm{~mm}$. The main exceptions which are presented are mainly the interferometers and the encoders.

Several sensors made with different technologies allow for sub-nanometric performances. ${ }^{6}$ Strain sensors, piezoelectric sensors, capacitive sensors, inductive sensors, encoders, and interferometers are the most common examples. Among all these technologies, the interferometry is obviously a good solution that combines both the good resolution and long stroke. ${ }^{7}$ Moreover, interferometers allow accuracy and traceability for displacement measurements. Their main drawbacks are their sensitivity to the medium index and their price that make them difficult to use in many applications.

Encoders may achieve similar performances to those obtained with a sensor based on the interferometry. For that purpose, Invar or Zerodur gratings are designed so that the periods are sub-micrometrics. In Ref. 8, encoders with nanometric resolution are presented. Moreover, they can be used to measure displacements over a few centimeters with very low and periodic non-linearities. However, for such performances, these sensors are expensive compared to other existing solutions. The compactness may be also an issue depending on the application and the available workspace. Fabry-Perot interferometers exhibit similar performances but suffer from a very complex tuning especially for fiber models. ${ }^{9}$

Other sensors are more affordable but do not necessarily meet the required performances like nanometric resolution and long range. For example, the resolution of inductive sensors is satisfying but not the accuracy. Capacitive sensors appear also as a good solution. However, a long range is difficult to achieve and the bandwidth is only of a few kilohertz. In Refs. 10-13, an integrated system consists of an emitting and a collecting fiber. High resolution and high bandwidth with a reasonable cost are made possible. But long terms drifts appear and lead to a lack of precision when a long term measurement is needed. In Refs. 14 and 15, two other systems are proposed. They are based on triangulation techniques. CCD and photodiode arrays are used but the bandwidth of those systems is limited. The compactness may be an issue again depending on the application.

This paper presents an interferometer with a fully embedded optical chip. In that way, the end-user should expect to set up his system without thinking of possible complex optical paths. In addition to its very good performances, this sensor demonstrates that low sensitivity to external disturbances and ease of use can be achieved. The interferometer uses an external telecom source, and integrated optical waveguides are inside the package. It partially explains why this sensor may be inexpensive compared to other interferometers. In Section II, 
the interferometer is presented with some technical details concerning the internal chip. In Section III, the experimental setup is described. In Section IV, we present and discuss the experimental results.

\section{INTERFEROMETRY SETUP}

\section{A. Description of the setup}

The optical chip has been designed initially for geophysical applications by the CEA/DAM (CEA: Commissariat à l'énergie atomique, DAM: Direction des applications militaires). The major concern was to optimize the noise level at low frequency because seismology phenomena and properties often appear in the sub-hertz range. The setup has been partially described in Ref. 16 where a first version has been built and tested. This prototype, made with macroscopic optical components, demonstrates very good noise results as low as $100 \mathrm{fm} \mathrm{Hz}^{-1 / 2} @ 8 \mathrm{~Hz}$. The second version has been built with the integrated waveguide technology to enhance the immunity to environmental disturbances, so that it is more compact and compatible with the vacuum environment. Evolutions have been also designed on optical paths inside the chip, for example to minimize the optical dead path. The version used in this paper corresponds to the third one without the evolution for vacuum compatibility.

The main optical system is fully integrated in an optical chip with an optical waveguide structure. The technology used for the chip is described in Ref. 17. The chip has been made by Teemphotonics, based on their knowledge of the integrated optics process. The waveguides are designed for the $1.55 \mu \mathrm{m}$ telecom window wavelength for convenience and limited cost. The optical setup is illustrated in Fig. 1(a).

An external laser source is connected through a fiber. The chip is very compact (see Fig. 2 for a picture with dimensions) and the package is represented in the right bottom insert of the figure. It is made of steel for vibration isolation and thermal homogeneity. All the inputs and the outputs are fibered. The output lens focuses the beam toward a mobile mirror and makes the system very easy to use. The lens is a collimating Grin lens. The beam is slightly diverging but the available range is a few centimeters as explained in the experimental part. The tuning of the system relies on the optimization of the retro-injected signal level in the chip.

The optical design presented in Fig. 1(b) is a mix between a Michelson interferometer and a Young interferometer. Indeed, the general architecture of the design is based on a Michelson interferometer since we use a reference arm with a fixed length and an arm with a varying length for measurement. Nevertheless, in order to get two outputs in quadrature, the interference between the two arms is implemented through a Young interferometer. The quadrature between $M_{1}$ and $M_{2}$ is assured when designing the chip. The distance $d_{\text {out }}$ between the waveguides at the output of the IFPZ is directly transferred from a photomask to the glass wafer through a photolithography step with a resolution better than $0.1 \mu \mathrm{m}$. $d_{\text {out }}$ is such that

$$
d_{\text {out }}=\frac{D}{4}=\frac{n \cdot \mu_{0} \cdot L}{4 d_{\text {in }}},
$$
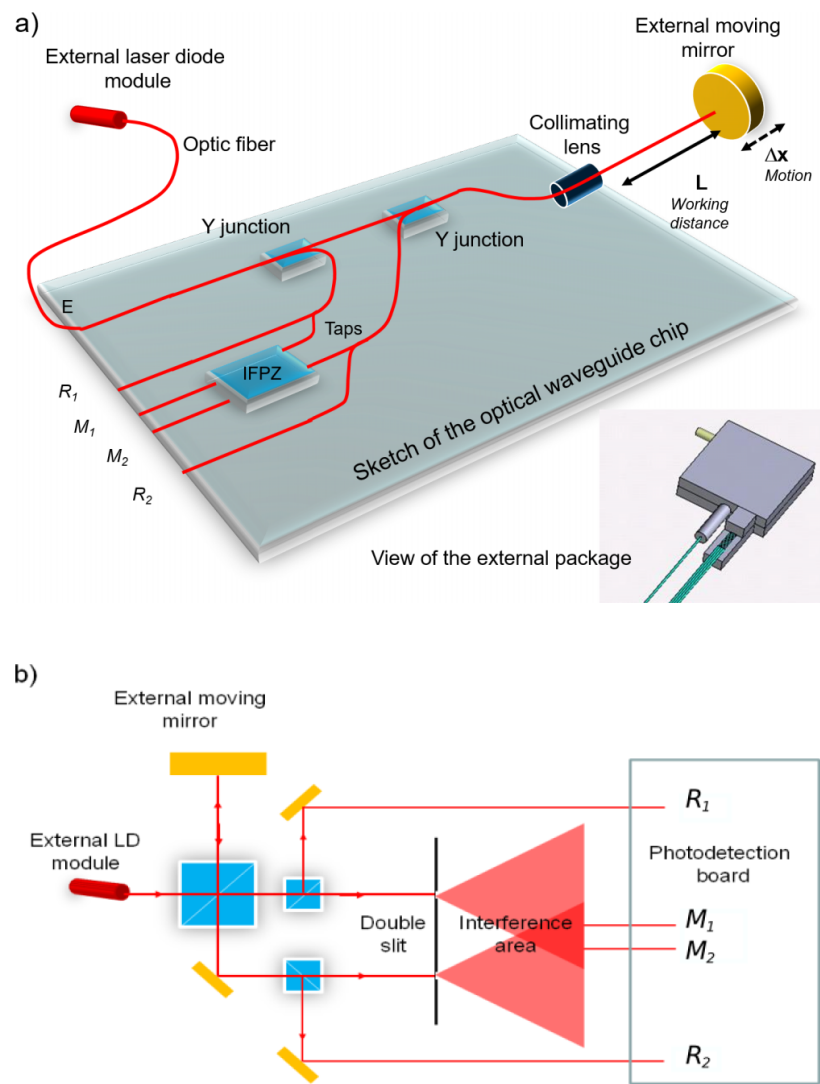

FIG. 1. Sketches of the optical chip. (a) optical representation with simplified optical paths of the beam in the chip; an external laser diode is used, $\mathrm{Y}$ junctions allow coupling between two paths, taps are junctions with only few percentages (1\% to 5\%) of coupling, and IFPZ is the Interference FreePropagation Zone; the four output signals are Reference $1\left(R_{1}\right)$ is a part of the incoming signal, Reference $2\left(R_{2}\right)$ is a part of the retro-injected signal, and Measurement $1\left(M_{1}\right)$ and Measurement $2\left(M_{2}\right)$ are the two quadrature signals at the output of the interference area; (b) principle of the interferometer and focus on the IPFZ area which is a double-slit structure with two outputs.

where $D$ is the spatial period of the sinusoidal interference pattern at the output of the IFPZ, $n$ is the effective index of the planar waveguide in the IFPZ area, $\mu_{0}$ is the operating wavelength in vacuum, $L$ is the length of the IFPZ, and $d_{i n}$ is

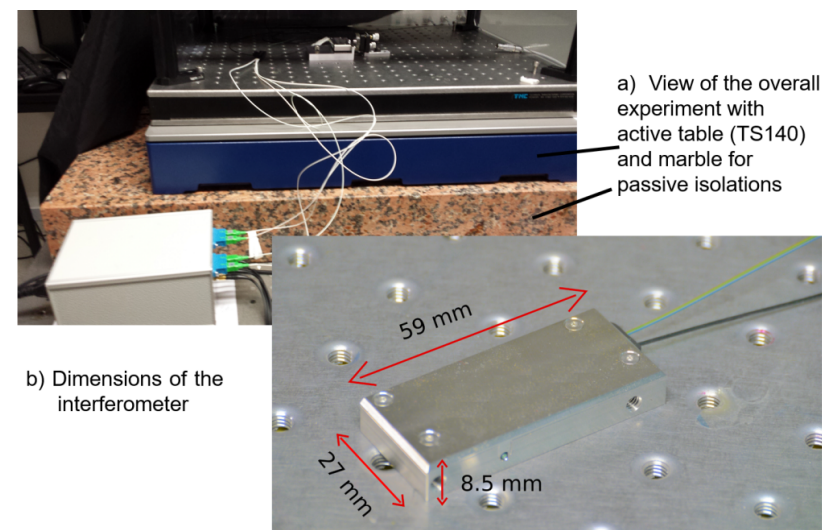

FIG. 2. Photography of the experiment. (a) The optical breadboard is fixed on an active table (piezoelectric systems, in blue on the photography) and placed on a marble which is also on an optical breadboard with four passive isolation feet; the overall system is protected from air flows by a plexiglas box and dark cover; (b) the interferometer is fixed on the breadboard in front of the mobile mirror (in static condition for the experiment but it can be driven by piezoelectric actuator if necessary). 
the distance between the waveguide at the input of the IFPZ area.

The optical loss in the IFPZ function is in the 12-14 dB range. Comparing to other solutions with lower optical loss, the implementation of an IFPZ allows a very low sensitivity to technological fluctuations of the manufacturing process.

The details of the principle with free beams and optical components can be found in Ref. 16. Basically, two Y junctions separate the beam along the measurements path and two taps provide the two reference arms $\left(R_{1}\right.$ and $\left.R_{2}\right)$ with a small amount of power (junction with $1 \%$ to $5 \%$ of coupling). At the output of the interference area, two measurements signals are available (sine-cosine outputs, $M_{1}$ and $M_{2}$ ). The four outputs are converted into voltages with the photodetector module (this is made with classical transimpedance structures). The optical interference between the reference path and the mobile path is made in the IFPZ area (Interference Free-Propagation Zone) which is a double-slit structure as illustrated in Fig. 1(b). $R_{1}$ allows compensations due to power fluctuations of the source, and $R_{2}$ allows compensations regarding the power of the retroinjected signal.

\section{B. Details about the position calculus}

First, each chip is calibrated before use. The Y junctions may be indeed slightly unbalanced and the insertion loss can also be different from one chip to another. The calibration aims to measure the $K_{i j}$ parameters that characterize the chip and coupling between the four outputs. The calibration can be made once or every time that the chip is manipulated because the parameters are dependent also on the insertion loss in the fibers that can be very sensitive. They are defined by Eqs. (2) and (3)

$$
\begin{gathered}
\left\{\begin{array}{l}
K_{11}=\frac{M_{1}}{R_{1}} \\
K_{12}=\frac{M_{2}}{R_{1}}
\end{array}\right. \\
\left\{\begin{array}{l}
K_{21}=\frac{\left(\frac{M_{1, \max }+M_{1, \min }}{2}\right)-R_{1} \times K_{11}}{R_{2}} \\
K_{22}=\frac{\left(\frac{M_{2, \max }+M_{2, \min }}{2}\right)-R_{1} \times K_{12}}{R_{2}},
\end{array}\right.
\end{gathered}
$$

where for Eq. (2) the retro-injected signal is blanked, and for Eq. (3), a displacement is imposed to obtain the sinusoidal response on $M_{1}$ and $M_{2} . M_{i, \max }$ and $M_{i, \min }$ represent the maximum and minimum values of the sinusoidal waveform. Typical values for $K_{11}, K_{12}, K_{21}$, and $K_{22}$ are around 0.6 to 0.7 .

Second, the $K_{i j}$ parameters are used to normalize the measurements with Eq. (4)

$$
\left\{\begin{array}{l}
M_{1, \text { norm }}(t)=\frac{M_{1}(t)-\left(K_{11} \cdot R_{1}(t)+K_{21} \cdot R_{2}(t)\right)}{2 \sqrt{K_{11} \cdot R_{1}(t) \times K_{21} \cdot R_{2}(t)}} \\
M_{2, \text { norm }}(t)=\frac{M_{2}(t)-\left(K_{12} \cdot R_{1}(t)+K_{22} \cdot R_{2}(t)\right)}{2 \sqrt{K_{12} \cdot R_{1}(t) \times K_{22} \cdot R_{2}(t)}},
\end{array}\right.
$$

where $t$ is the time.
The normalization allows to be more robust to power fluctuations of the source (thanks to $R_{1}$ ) and of the retroinjected signal (thanks to $R_{2}$ ).

Once the normalized signals are obtained, it allows the estimation of the classical Lissajous ellipse, described by Eq. (5)

$$
\left\{\begin{array}{l}
M_{1, \text { norm }}(t)=A_{0} \cdot \cos (\phi(t))+X_{0} \\
M_{2, \text { norm }}(t)=B_{0} \cdot \sin \left(\phi(t)+\Phi_{0}\right)+Y_{0}
\end{array},\right.
$$

where $A_{0}, B_{0}, X_{0}, Y_{0}$, and $\Phi_{0}$ are the parameters of the ellipse.

Once the ellipse is fully estimated, the measurement of the output phase $\phi(t)$ of the interferometer is derived from Eq. (5) and finally the output expression of the displacement is

$$
\Delta x(t)=\frac{\lambda \cdot \Delta \phi(t)}{4 \pi},
$$

where $\lambda$ is the wavelength of the source, $\Delta \phi$ is the phase variation measured with the Lissajous method, and $\Delta x$ is the displacement of the mobile mirror.

The computation of the phase is made in real-time and the bandwidth of the interferometer output is actually limited by the sample frequency of the data acquisition board.

The only limitation on the computing is the maximum displacement speed. The maximum phase shift between two acquisitions should be $\pi$ because of the indeterminate forms and Shannon criteria. For a sample frequency of $30 \mathrm{kHz}$ and a wavelength of $1.55 \mu \mathrm{m}$, the maximum speed is almost $10 \mathrm{~mm} \mathrm{~s}^{-1}$.

\section{EXPERIMENTAL SETUP}

The aim of this work is mainly to evaluate the interferometer in quasi-static conditions or with very small displacements in order to estimate the long term performances. Therefore, a particular care is attended to monitor the environmental conditions of the experiment. Fig. 2 represents a photography of the whole setup. The interferometer is hardly fixed on a breadboard in front of a mobile mirror which can be tuned for retro-injection. The breadboard is placed on an active table (active piezoelectric system TS140 from Table Stable) that isolates from building vibrations with a relatively low bandwidth. The table is placed on a marble which is fixed on a second optical breadboard with four passive feet. The experiment is protected from air flow disturbances with a Plexiglas box covered with a dark sheet to eliminate the light perturbations. There is no active temperature regulation but this parameter is monitored.

The laser source is a telecom laser module (ORION Rio0085-1-00-1) with a wavelength value of $1542.135 \mathrm{~nm}$ (1). The uncertainty and the influence of the wavelength will be discussed in the sequel. The source is connected to the interferometer module with an optical fiber and the power can be controlled with a LabVIEW software. The mean power at the output of the optical chip for the useful moving arm is around $140 \mu \mathrm{W}$.

The four output signals can be sampled by different systems of Analog-to-Digital Converter boards (ADC). The first one is a 4 channel-fully synchronized-with 24 bits 
resolution (NI PCI-4472) which is a very low noise sample system dedicated to noise sound analysis (with a maximal sampling frequency of $102.4 \mathrm{kHz}$ ). The second one is a 4 channel-fully synchronized-16 bits resolution (NI PXI$6143)$ with a higher sampling frequency $(250 \mathrm{kHz})$.

The four signals are acquired with a LabVIEW software and saved on a computer for $a$ posteriori calculus. The calculus includes low-pass filtering, statistical analysis, and calculus of the ellipse. The position is then calculated and recorded in data files for the post-treatment analysis. The sample frequency and parameters of the filters are programmable.

The external mobile mirror can be either a simple mirror mounted on tilt mechanical stages for tuning or a mirror mounted on a specific mechanical system. The mechanical stage can be driven with a piezoelectric actuator along the measurement axis.

\section{RESULTS AND DISCUSSIONS}

\section{A. Static evaluation}

Once the experiment is fully operational, the interferometer is tuned by optimizing the retro-injected signal. $R_{2}$ signal is optimized as well as the contrast on $M_{1}$ and $M_{2}$. When the interferometer is correctly tuned, the box is closed and the measurement recorded during a calm period (in general during the night or the week-end in order to minimize the human activity perturbations). The first curves have been made with the 24 bits ADC system.

Several distances between the output collimating lens and mobile mirror have been tested. Nominal conditions are $1 \mathrm{~cm}$ and $4 \mathrm{~cm}$ but several sets of distance have been tested (up to $6 \mathrm{~cm}$ ) and results are very similar. Hereafter, we present results for nominal conditions.

Fig. 3 shows measurements as a function of time over $10 \mathrm{~min}$. The sampling frequency is $30 \mathrm{kHz}$ but a mean is calculated over 10 points to make the figure clearer. Therefore,

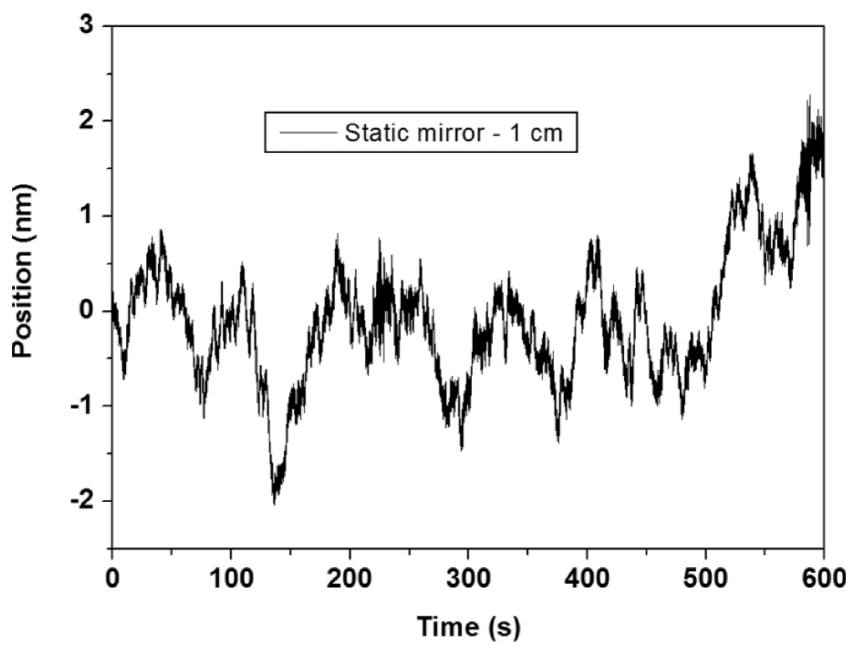

FIG. 3. Measurements of the relative position of the mirror in a static configuration (the working distance is $1 \mathrm{~cm}$ ). The sample frequency is $30 \mathrm{kHz}$ and a mean value is calculated with 10 points before plotting. The drift is due to residual environmental perturbations but one can see that over $10 \mathrm{~min}$, the residual perturbations remain reasonable.
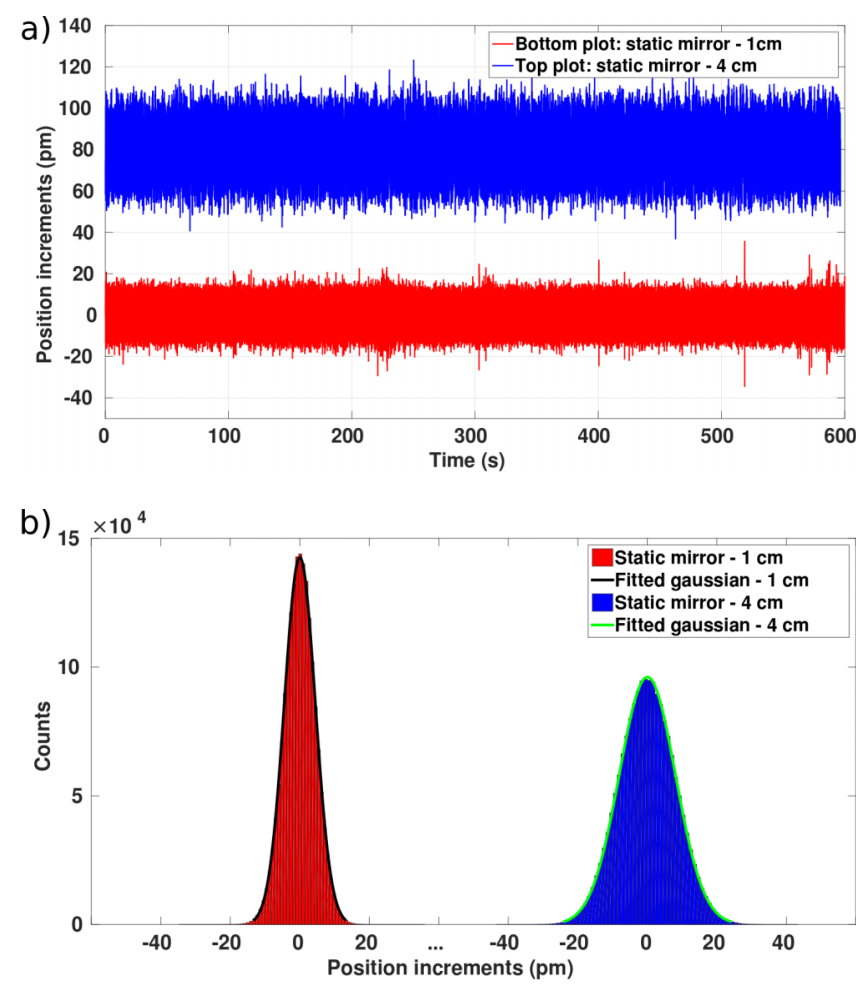

FIG. 4. Plot of differential measurements. The experiment has been made for two distances $(1 \mathrm{~cm}$ and $4 \mathrm{~cm}$ ) and plots have been separated for clarity. The differential measurements are the difference between the positions at time $t$ and $t+T_{s}$. Top view (a) is time dependent and bottom view (b) is the distribution with a Gaussian profile.

the plotting frequency is $3 \mathrm{kHz}$. Of course, over $10 \mathrm{~min}$, a remaining drift appears and small perturbations of a few nanometers are visible.

It is quite impossible to be fully insensitive to the environment. In order to estimate the potential of the instrument, a differential measurement has been plotted in Fig. 4. The part (a) presents the data with respect to the time.

Two working distances have been used and separated on the plot for clarity. The sampling frequency is $30 \mathrm{kHz}$ and a mean value is calculated with 10 samples so that the position is updated at a frequency $f_{s}=3 \mathrm{kHz}$ (the period $T_{s}=1 / f_{s}$ can be modified). After this step, a difference is computed. Each point of the plot is the result of the difference between the mean position at time $t$ and $t+T_{s}$. The aim of this differential measurement is to avoid all environmental perturbations and to only measure the limits of the optical chip associated with the instrumentation chain. The bottom curves represent the respective histograms with Gaussian fits.

One can see that the remaining noise is Gaussian with a standard deviation of $4.5 \mathrm{pm}$ and $8 \mathrm{pm}$, respectively, for $1 \mathrm{~cm}$ and $4 \mathrm{~cm}$ working distances. The performances for the working distance of $4 \mathrm{~cm}$ are degraded because of the output lens. The beam is collimated for a few centimeters and is slightly diverging for higher distances. Consequently, the retro-injected signal is lower for longer distance as well as the signal to noise ratio. However, the performances are below the nanometer level which makes the interferometer competitive compared to the commercial apparatus. Taking into account the present output lens, we have made some tests 
a)

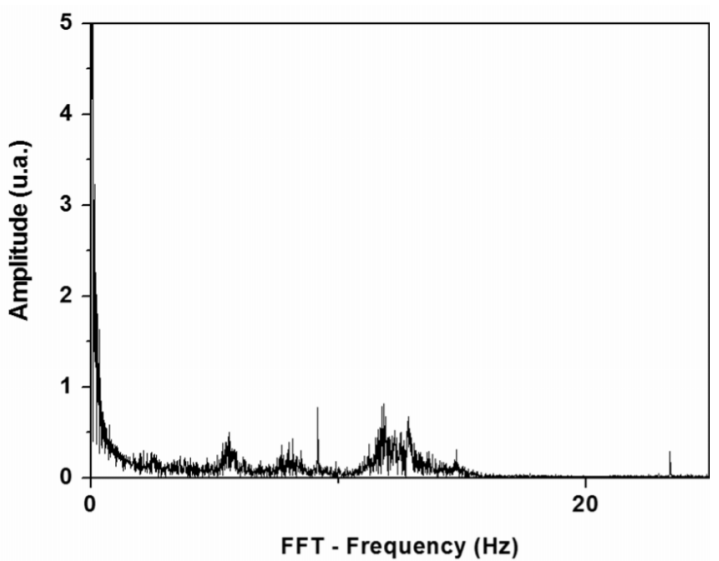

b)

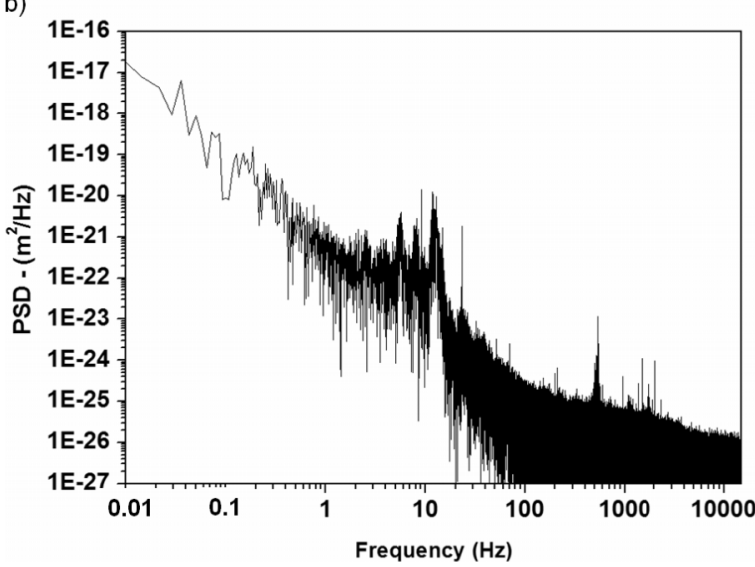

FIG. 5. (a) Fast Fourier Transform (FFT-top view) and (b) power spectral density (PSD - bottom view) of the vibration measurements on the mirror in a static configuration. On the FFT, we can clearly see the remaining vibration of the building around $10 \mathrm{~Hz}$ and the flicker noise below $2 \mathrm{~Hz}$. The PSD noise level is around $400 \mathrm{fm} \mathrm{Hz}^{-1 / 2} @ 100 \mathrm{~Hz}$ and seems to converge toward a noise level of $100 \mathrm{fm} \mathrm{Hz}^{-1 / 2} @ 10 \mathrm{kHz}$.

up to $6 \mathrm{~cm}$ stroke but it could be enhanced by changing the lens.

Fig. 5 represents the Fast Fourier Transform (FFT) and the Power Spectral Density (PSD) of the measurements. The FFT is used as a tool to check that the remaining noise is mainly in the low frequency spectrum range. It is due to the vibrations of the building which are very hard to cancel. The remaining free oscillating frequencies appear around $5 \mathrm{~Hz}, 9 \mathrm{~Hz}$, and $12 \mathrm{~Hz}$ and of course some noise in a very low frequency.

On the PSD plot, the frequency range covered by the measurements is quite large, from $0.01 \mathrm{~Hz}$ to $15 \mathrm{kHz}$. We can see also the noise due to oscillating frequencies around $10 \mathrm{~Hz}$. The PSD noise level is around $400 \mathrm{fm} \mathrm{Hz}^{-1 / 2} @ 100 \mathrm{~Hz}$ and converges to the level of $10^{-26} \mathrm{~m}^{2} / \mathrm{Hz} @ 10 \mathrm{kHz}$, which corresponds to $100 \mathrm{fm} \mathrm{Hz}^{-1 / 2}$ @ $10 \mathrm{kHz}$.

In complement to the results obtained with an analysis for high frequencies, a longtime estimation has been performed. Several sets of measurements have been recorded, either over short time with $30 \mathrm{kHz}$ sampling frequency or with mid and long term range with $1 \mathrm{kHz}$ and $100 \mathrm{~Hz}$ sampling frequencies. The Allan standard deviation $\sigma_{y}{ }^{18}$ has been estimated for different integration times $\tau$. Note that the residual oscillation noise due to the building environment has not been compensated because it would have required

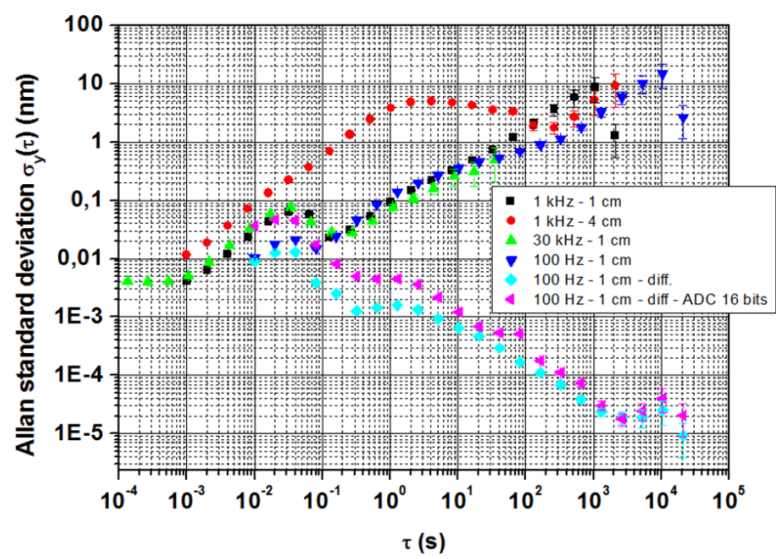

FIG. 6. Allan standard deviation depending on the integration time $\tau$. Several curves have been plotted to explore different time domains. The very short term standard deviation (up to $20 \mathrm{~ms}$ ) remains below $10 \mathrm{pm}$. The fourth top curve ascends up to $100 \mathrm{pm}$ or even several $\mathrm{nm}$ because of the environmental perturbations that influence the long term $(>100 \mathrm{~s})$ and the two bottom curves are calculated on differential positions to explore the potential of the long term stability.

band-pass filtering and we did not want to suppress the useful information. Fig. 6 represents the plots of the Allan standard deviation depending on the integration time $\tau$ from $3 \times 10^{-4} \mathrm{~s}$ to $20000 \mathrm{~s}$ (one night-long and more).

For the working distance of $1 \mathrm{~cm}$, the very short time estimation of the Allan standard deviation remains mainly below $10 \mathrm{pm}$ and below $1 \mathrm{~nm}$ for integration time up to tens of seconds (black square, green triangle, and blue triangle curves). We can see again that the signal to noise ratio is lower and that the measurements can be degraded for a higher working distance. The bump between $10^{-2} \mathrm{~s}$ and $10^{-1} \mathrm{~s}$ corresponds to the remaining noise of the building at a few hertz and the level may depend on the moment when the records are performed. The experiments made for a long duration (1 h-long or more) lead to a drift of several nanometers. This is mainly due to the environmental conditions like the temperature and the mechanical deformations.

In order to estimate the potential of the apparatus, the techniques described in Fig. 4 are used. The differential measurements are calculated to avoid drifts and the Allan standard deviation is plotted. It corresponds to the two bottom curves (blue diamonds and purple triangles). Acquisition has been made either with the 24 bits ADC system or the 16 bits ADC system. The long time estimation $(>1 \mathrm{~h})$ seems to converge toward the level of $20 \mathrm{fm}$.

It is then likely that the performances could be better for long time integration time with better environmental conditions: vacuum condition or air conditioning, a better active anti-vibration table, and no residual mechanical oscillation.

\section{B. Dynamic evaluation}

The mobile mirror is now driven by a piezoelectric actuator along the axis of the interferometer. The aim of this part is to evaluate the dynamic performances of the whole system. To avoid the natural residual vibration detected before $(5,12$, and $9 \mathrm{~Hz}$ mainly), the piezoelectric actuator is driven 
a)

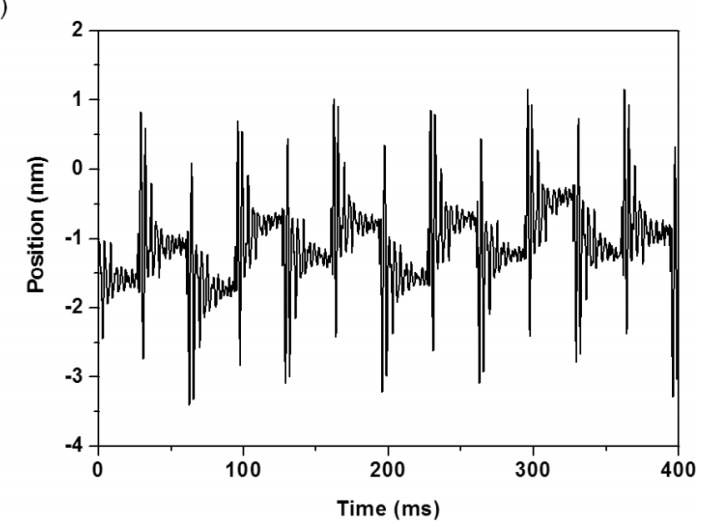

b)

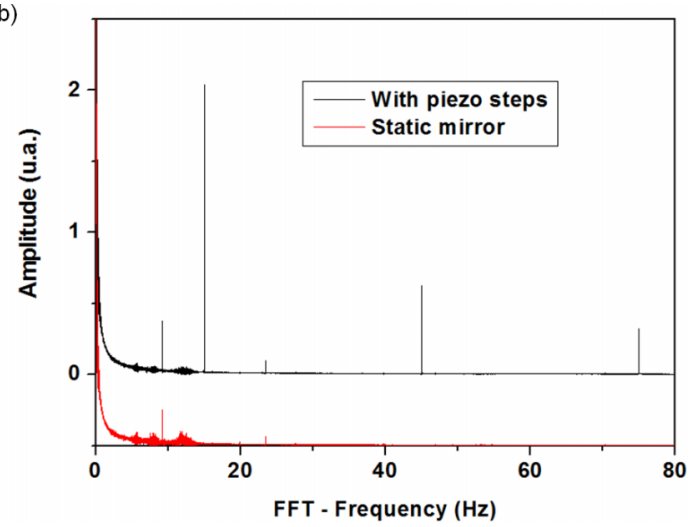

FIG. 7. (a) Steps of $0.5 \mathrm{~nm} @ 15 \mathrm{~Hz}$ (sampling frequency of $30 \mathrm{kHz}$ ). The piezoelectric is in an open-loop control mode and the measurement resolution of the interferometer is obviously below $0.1 \mathrm{~nm}$. Residual mechanical oscillations appear on each front; (b) FFT without steps (red curve downside, with the natural oscillation frequency around $9 \mathrm{~Hz}$ ) and with the steps (black curve upside with $15 \mathrm{~Hz}$ line and the odd harmonics $45 \mathrm{~Hz}$ and $75 \mathrm{~Hz}$ ).

with a square waveform (a frequency of $15 \mathrm{~Hz}$ and a magnitude of a few millivolts).

Fig. 7(a) shows the results of time measurements with a sampling frequency of $30 \mathrm{kHz}$. We clearly see the steps with an amplitude of $0.5 \mathrm{~nm}$. However, the piezoelectric actuator is in an open-loop control mode and the steps are thus very disturbed, with second order oscillations on the front edges.

Fig. 7(b) is the FFT view. Two curves have been plotted (separated for clarity). The top one (black) is obtained when the piezoelectric actuator is driven with the waveform. The bottom one is obtained when no signal is applied to the actuator. The free oscillation around $9 \mathrm{~Hz}$ is due to the building in both cases. On the top curve, the signal excitation frequency of $15 \mathrm{~Hz}$ is present as well as the odd harmonics (45 and $75 \mathrm{~Hz})$.

\section{Discussion}

This system reaches very good performances when noise level is concerned as demonstrated in experiments. It is difficult to compare exactly the noise performances between all the systems presented in the literature because, at very low level, the environment is overriding. Nevertheless, power spectral density level reaches typically $20 \mathrm{pm} \mathrm{Hz}^{-1 / 2} @ 100 \mathrm{~Hz}$ for strain sensors, ${ }^{6} 1 \mathrm{pm} \mathrm{Hz}^{-1 / 2} @ 100 \mathrm{~Hz}$ for capacitive sensors for $10 \mu \mathrm{m}$ stroke or mostly $40 \mathrm{pm} \mathrm{Hz}^{-1 / 2} @ 100 \mathrm{~Hz}$ for capacitive sensors for $100 \mu \mathrm{m}$ stroke, ${ }^{19} 50 \mathrm{fm} \mathrm{Hz}^{-1 / 2} @$ $100 \mathrm{~Hz}$ for piezoelectric strain sensors for few $\mu \mathrm{m}$ stroke, ${ }^{6}$ and $10 \mathrm{pm} \mathrm{Hz}-1 / 2 @ 100 \mathrm{~Hz}$ for integrated thermal position sensors for $100 \mu \mathrm{m}$ stroke. ${ }^{20}$ Our sensor, with a $400 \mathrm{fm}$ $\mathrm{Hz}^{-1 / 2} @ 100 \mathrm{~Hz}$, is thus highly competitive compared to other sensors in terms of noise. Furthermore, these sensors are always dedicated to applications that require a stroke of a few micrometers. They have also a low bandwidth compared to the potential of systems based on the interferometry. For these sensors, the performances can be better for lower frequencies when the interferometry is very sensitive to the environment. It is very difficult to compare the PSD between commercial interferometers but, as far as we know, the noise level is very good. A recent example of closed-loop phase-locked prototype ${ }^{21}$ shows noise level around $100 \mathrm{pm} \mathrm{Hz}^{-1 / 2} @ 100 \mathrm{~Hz}$ and $100 \mathrm{fm} \mathrm{Hz}^{-1 / 2} @ 10 \mathrm{kHz}$.

In the domain of compact and integrated interferometer, many scientists have prospected for ultra-high performances. Fiber optic interferometers are highly sensitive to the environment because of the sensitivity of the fiber optic. FabryPerot fibered interferometers usually reach few $\mathrm{pm} \mathrm{Hz}^{-1 / 2} .^{22}$ The Atomic Force Microscopy (AFM) community has also developed several dedicated interferometers to sense the cantilever and obtained outstanding performances near of the shot noise, few $\mathrm{fm} \mathrm{Hz}{ }^{-1} .{ }^{23-30}$ Nevertheless, these systems are usually not suitable for applications that require a long stroke. $^{31-40}$

The environmental conditions are also critical for the system behavior and the performances. The interferometer that we have presented was first designed for a seismometer. Experiments were performed in a dedicated deep underground station and $100 \mathrm{fm} \mathrm{Hz}^{-1 / 2} @ 8 \mathrm{~Hz}$ was reached. However, we did not succeed to reproduce these ideal conditions in our laboratory. The noise isolation is not the only issue for environmental conditions. The temperature fluctuation is an important factor since it changes the refractive index of air (around $10^{-6}$ in relative value for $1{ }^{\circ} \mathrm{C}$ change). The fluctuation of the wavelength of the source is also a parameter which influences the long term measurements. For the RIO source that we are using, the Allan standard deviation of the wavelength is limited to $10^{-8}$ over thousands of seconds (15 fm@1.55 $\mu \mathrm{m})$. Moreover, the source is also affected by the temperature and it limits the actual displacement range. For example, with an uncertainty of $1{ }^{\circ} \mathrm{C}$, the relative uncertainty of the wavelength is $3 \times 10^{-7}$. The displacement range is then equal to the resolution divided by $3 \times 10^{-7}$. As a consequence, the maximum range is $3 \mathrm{~mm}$ if $1 \mathrm{~nm}$ of accuracy is required. This may be problematic for applications like multiscale scanning when the displacement range is a concern.

To improve the system, we plan to develop a chip which may be used with a red source instead of the infrared one. That would allow the use of ultrastable sources (red lasers, for example). It could be also easier to set up especially for applications like AFM scanning when the focus of the beam on the cantilever is difficult. A future version will be also vacuum compatible, allowing the integration in a vacuum chamber (chambers of electronic microscopes, for example). 


\section{ACKNOWLEDGMENTS}

This research was supported by the French research ministry (FUI2013 program through a project called MULTISS) and the Conseil départemental des Yvelines.

Authors are to be grateful to S. Olivier from DGA/DAM for his original help about all the hardware and software packages. Authors thank also Frédéric Mourgues for mechanical support.

${ }^{1}$ M. Miles, "Probing the nanoworld," Nanotechnology 20, 430208 (2009).

${ }^{2}$ J. Brugger, "Nanotechnology impact on sensors," Nanotechnology 20, 430206 (2009).

${ }^{3}$ C. Pacoret and S. Régnier, "A review of haptic optical tweezers for an interactive microworld exploration," Rev. Sci. Instrum. 84, 081301 (2013).

${ }^{4}$ L. Chassagne, S. Blaize, P. Ruaux, S. Topcu, P. Royer, Y. Alayli, and G. Lérondel, "Multi-scale scanning probe microscopy," Rev. Sci. Instrum. 81, 086101 (2010).

${ }^{5}$ G. Lérondel, A. Sinno, L. Chassagne, S. Blaize, P. Ruaux, A. Bruyant, S. Topçu, P. Royer, and A. Alayli, "Enlarged near-field optical imaging," J. Appl. Phys. 106, 044913 (2009).

${ }^{6}$ A. J. Fleming, "A review of nanometer resolution position sensors: Operation and performance," Sens. Actuators, A 190, 106-126 (2013).

${ }^{7}$ N. Bobroff, "Recent advances in displacement measuring interferometry," Meas. Sci. Technol. 4, 907 (1993).

${ }^{8}$ A. Yacoot and N. Cross, "Measurement of picometer non-linearity in an optical grating encoder using x-ray interferometry,” Meas. Sci. Technol. 14, 148-152 (2003).

${ }^{9}$ M. Durand, J. Lawall, and Y. Wang, "High-accuracy Fabry-Perot displacement interferometry using fiber lasers," Meas. Sci. Technol. 22, 094025 (2011).

${ }^{10}$ M. Kim, W. Moon, E. Yoon, and K.-R. Lee, "A new capacitive displacement sensor with high accuracy and long-range," Sens. Actuators, A 130-131, 135-141 (2006).

${ }^{11}$ M. Yasin, S. W. Harun, K. Karyono, and H. Ahmad, "Fiber optic displacement sensor using a multimode bundle fiber," Microwave Opt. Technol. Lett. 50, 661-663 (2008).

${ }^{12}$ R. Dib, Y. Alayli, and P. Wagstaff, "A broadband amplitude-modulated fiber optic vibrometer with nanometric accuracy," Measurement 35, 211-219 (2004).

${ }^{13}$ A. Khiat, F. Lamarque, C. Prelle, P. Phouille, M. Leester-Schädel, and S. Büttganbach, "Two-dimension fiber optic sensor for high-resolution and long-range linear measurements," Sens. Actuators, A 158, 43-50 (2010).

${ }^{14}$ L. Perret, L. Chassagne, S. Topcu, P. Ruaux, B. Cagneau, and Y. Alayli, "Fiber optics sensor for sub-nanometric displacement and wide bandwidth systems," Sens. Actuators, A 165, 189-193 (2011).

${ }^{15}$ A. Missoffe, S. Topcu, P. Ruaux, B. Cagneau, and Y. Alayli, "New simple optical sensor: From nanometer resolution to centimeter displacement range," Sens. Actuators, A 176, 46-52 (2012).

${ }^{16}$ D. Ponceau, P. Millier, and S. Olivier, "Subnanometric Michelson interferometry for seismological applications," Proc. SPIE 7003, 70030U (2008).
${ }^{17}$ F. Malbet, P. Kern, I. Schanen-Duport, J.-P. Berger, K. Rousselet-Perraut, and P. Benech, Astron. Astrophys., Suppl. Ser. 138, 135 (1999).

${ }^{18}$ D. W. Allan, H. Hellwig, and D. J. Glaze, "An accuracy algorithm for anatomic time scale," Metrologia 11, 133 (1975).

${ }^{19}$ A. J. Fleming, A. G. Wills, and S. O. R. Moheimani, "Sensor fusion for improved control of piezoelectric tube scanners," IEEE Trans. Control Syst. Technol. 15(6), 1265-6536 (2008).

${ }^{20}$ Y. Zhu, A. Bazaei, S. O. R. Moheimani, and M. Yuce, "Design modeling andcontrol of a micromachined nanopositioner with integrated electrothermal actuation and sensing," J. Microelectromech. Syst. 20(3), 711-719 (2011).

${ }^{21}$ A. J. Fleming and B. Routley, "A closed-loop phase-locked interferometer for wide bandwidth position sensing," Rev. Sci. Instrum. 86, 115001 (2015).

${ }^{22}$ K. Karrai and P. Braun, "Miniature long-range laser displacement sensor," in Proceedings Actuator (Bremen, Germany, 2010), pp. 285-288.

${ }^{23}$ D. Rugar, H. J. Mamin, R. Erlandsson, J. E. Stern, and B. D. Terris, Rev. Sci. Instrum. 59, 2337-2340 (1988).

${ }^{24}$ B. W. Hoogenboom, P. L. T. M. Frederix, J. L. Yang, S. Martin, Y. Pellmont, M. Steinacher, S. Zäch, E. Langenbach, H.-J. Heimbeck, A. Engel, and H. J. Hug, "A Fabry-Perot interferometer for micrometer-sized cantilevers," Appl. Phys. Lett. 86, 074101 (2005).

${ }^{25}$ H. I. Rasool, P. R. Wilkinson, A. Z. Stieg, and J. K. Gimzewski, “A low noise all-fiber interferometer for high resolution frequency modulated atomic force microscopy imaging in liquids," Rev. Sci. Instrum. 81, 023703 (2010).

${ }^{26}$ L. Bellon, S. Ciliberto, H. Boubaker, and L. Guyon, "Differential interferometry with a complex contrast," Opt. Commun. 207, 49-56 (2002).

${ }^{27}$ Y. Martin, C. C. William, and H. K. Wickramasinghe, "Atomic force microscope-force mapping and profiling on a sub 100- $\AA$ scale," J. Appl. Phys. 61(10), 4723 (1987).

${ }^{28}$ A. J. den Boef, "Scanning force microscopy using a simple low-noise interferometer," Appl. Phys. Lett. 55, 439 (1989).

${ }^{29}$ R. M. Delarue, R. F. Humphryes, L. M. Mason, and E. A. Ash, Proc. IEEE 119, 117 (1972)

${ }^{30}$ P. Paolino, F. A. A. Sandoval, and L. Bellon, "Quadrature phase interferometer for high resolution force spectroscopy,” Rev. Sci. Instrum. 84, 095001 (2013).

${ }^{31}$ D. Rugar, H. J. Mamin, and P. Guethner, "Improved fiber-optic interferometer for atomic force microscopy," Appl. Phys. Lett. 55, 2588-2590 (1989).

${ }^{32}$ C. Schönenberger and S. F. Alvarado, "A differential interferometer for force microscopy,” Rev. Sci. Instrum. 60, 3131-3134 (1989).

${ }^{33}$ P. J. Mulhern, T. Hubbard, C. S. Arnold, B. L. Blackford, and M. H. Jericho, Rev. Sci. Instrum. 62, 1280-1284 (1991).

${ }^{34}$ G. Meyer and N. M. Amer, Appl. Phys. Lett. 53, 2400 (1988).

${ }^{35}$ C. A. J. Putman, B. G. de Grooth, N. F. van Hulst, and J. Greve, J. Appl. Phys. 72, 6-12 (1992).

${ }^{36}$ M. G. L. Gustafson and J. Clarke, J. Appl. Phys. 76, 172-181 (1994).

${ }^{37}$ T. Fukuma, M. Kimura, K. Kobayashi, K. Matsushige, and H. Yamada, Rev. Sci. Instrum. 76, 053704 (2005).

${ }^{38}$ T. Fukuma and S. P. Jarvis, Rev. Sci. Instrum. 77, 043701 (2006).

${ }^{39}$ T. Fukuma, Rev. Sci. Instrum. 80, 23707 (2009).

${ }^{40}$ R. Enning, D. Ziegler, A. Nievergelt, R. Friedlos, K. Venkatarani, and A. Stemmer, Rev. Sci. Instrum. 82, 043705 (2011). 